\title{
Glutamina y antioxidantes en pacientes críticos con lesión renal aguda en terapia de reemplazo renal
}

\section{Glutamine and antioxidants in critically ill patients with acute kidney injury on renal replacement therapy}

\section{Francisco Gerardo Yanowsky-Escatell ${ }^{a}{ }^{a}$, Leonardo Pazarín-Villaseñor ${ }^{a}$, Jorge Andrade-Sierra ${ }^{a}$, Miguel Ángel Zambrano-Velarde ${ }^{a}$, Francisco Martín Preciado-Figueroa ${ }^{a}$}

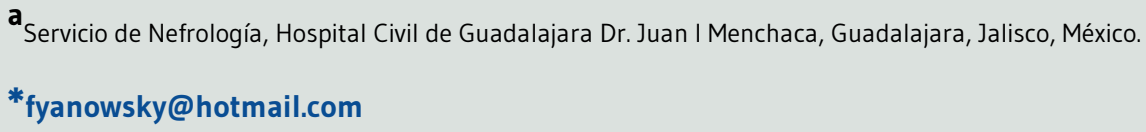

Recidido el 17 de enero de 2017; aceptado el 20 de marzo de 2017.

\section{CITA}

Yanowsky-Escatell FG, Pazarín-Villaseñor L, Andrade-Sierra J, Zambrano-Velarde MA, Preciado-Figueroa FM. Glutamina y antioxidantes en pacientes críticos con lesión renal aguda en terapia de reemplazo renal. Rev Esp Nutr Hum Diet. 2017; 21(1): 1-2. doi: 10.14306/renhyd.21.1.342

La lesión renal aguda es un síndrome que se caracteriza por un rápido deterioro (horas o días) de la función renal, es frecuente en pacientes críticos y se asocia con complicaciones severas. Las principales consecuencias en esta condición son la acumulación de productos de desecho, electrolitos y fluidos, así como de reducción en la inmunidad y disfunción de órganos no renales ${ }^{1}$.

En pacientes críticos con lesión renal aguda es requerida la terapia de reemplazo renal (TRR), lo que contribuye a una extensa perdida de micronutrientes incluidos la glutamina (GIn) y antioxidantes. Actualmente los tipos de TRR más utilizados para estos pacientes son la hemodiálisis intermitente para unidades renales y la terapia de reemplazo renal continua (TRRC) como hemofiltración venovenosa continua para unidades de cuidados intensivos. Otro tipo de terapia utilizada es la diálisis sostenida de baja eficiencia, siendo la
TRRC la que mayor pérdida de micronutrientes presenta de las tres².

GIn y antioxidantes son micronutrientes inmunomoduladores que pueden intervenir en la respuesta inflamatoria, estrés oxidativo y función inmune. La recomendación para recibir estos suplementos está basada en los bajos niveles plasmáticos que presentan los pacientes críticos en las unidades de cuidados intensivos. Sin embargo, los beneficios clínicos de su suplementación se limitan a estudios pequeños, antiguos y en centros únicos ${ }^{3}$. Su uso en pacientes críticos con lesión renal aguda en TRR ha sido poco estudiado.

Berg y Cols. observaron la pérdida de GIn que existe en pacientes con falla multiorgánica en TRRC, además, que la provisión parenteral de la misma incrementa sus concentraciones plasmaticas ${ }^{4}$. Otros estudios han demostrado las pérdidas significativas y balances negativos de antioxidantes 
que se presentan durante la TRRC ${ }^{5,6}$. A pesar de que estos trabajos concluyeron que la pérdida de estos micronutrientes durante esta terapia sugiere mayor necesidad de suplementación ${ }^{4-6}$. En el análisis post hoc del estudio REDOXS se observó que la mortalidad a los 28 días fue significativamente mayor en el grupo de pacientes con disfunción renal que recibió suplementos de GIn y antioxidantes frente al grupo placebo. El efecto negativo de este tratamiento fue atenuado por el inicio de la TRR después de la aleatorización, pero cuando estos micronutrientes se combinaron el efecto fue deletéreo (OR=3,07; IC95\%:1,24-7,59)7.

En un estudio reciente en pacientes críticos con lesión renal aguda en TRRC se concluyó que los niveles séricos de antioxidantes no influyeron sobre la mortalidad, estancia prolongada tanto en la unidad de cuidados intensivos como hospital, y en duración de la $\operatorname{TRR}^{8}$. Oh y Cols. en su artículo de revisión mencionan que aún se desconoce si las pérdidas de micronutrientes son clínicamente significativas o si su suplementación mejoraría los resultados en estos pacientes². Además, las guías internacionales de la European Society for Clinical Nutrition and Metabolism (ESPEN) y la American Society for Parenteral and Enteral Nutrition (ASPEN) para el soporte nutricional en pacientes con lesión renal aguda carecen del suficiente nivel de evidencia científica para recomendar su suplementación ${ }^{9,10}$.

En la actualidad, los beneficios de la suplementación de GIn y antioxidantes en pacientes críticos con lesión renal aguda en TRR son limitados. Las pérdidas de estos micronutrientes durante la TRR ha sido observada, pero la suplementación de los mismos no parece ser benéfica, sino más bien perjudicial en presencia de disfunción renal. Por lo cual consideramos que no son aconsejables y que tanto la seguridad como eficacia de estos suplementos debe ser demostrada en estos pacientes.

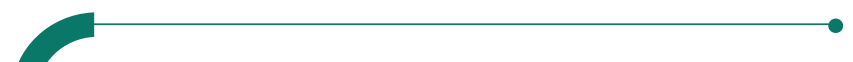

\section{CONFLICTO DE INTERESES}

Los autores expresan que no hay conflictos de interés al redactar el manuscrito.

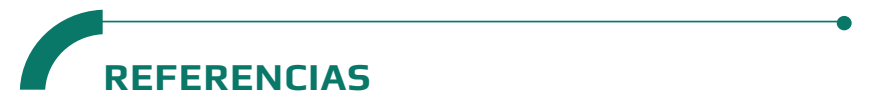

(1) Ostermann M, Joannidis M. Acute kidney injury 2016: diagnosis and diagnostic workup. Crit Care. 2016; 20(1): 299.

(2) Oh WC, Gardner DS, Devonald MAJ. Micronutrient and amino acid losses in acute renal replacement therapy. Curr Opin Clin Nutr Metab Care. 2015; 18(6): 593-8.

(3) van Zanten ARH. Glutamine and antioxidants: status of their use in critical illness. Curr Opin Clin Nutr Metab Care. 2015; 18(2): 179-86.

(4) Berg A, Norberg A, Martling C-R, Gamrin L, Rooyackers O, Wernerman J. Glutamine kinetics during intravenous glutamine supplementation in ICU patients on continuous renal replacement therapy. Intensive Care Med. 2007; 33(4): 660-6.

(5) Berger MM, Shenkin A, Revelly J-P, Roberts E, Cayeux MC, Baines $M$, et al. Copper, selenium, zinc, and thiamine balances during continuous venovenous hemodiafiltration in critically ill patients. Am J Clin Nutr. 2004; 80(2): 410-6.

(6) Story DA, Ronco C, Bellomo R. Trace element and vitamin concentrations and losses in critically ill patients treated with continuous venovenous hemofiltration. Crit Care Med. 1999; 27(1): 220-3.

(7) Heyland DK, Elke G, Cook D, Berger MM, Wischmeyer PE, Albert $M$, et al. Glutamine and antioxidants in the critically ill patient: a post hoc analysis of a large-scale randomized trial. JPEN J Parenter Enteral Nutr. 2015; 39(4): 401-9.

(8) Kritmetapak K, Peerapornratana S, Srisawat N, Somlaw N, Lakananurak N, Dissayabutra T, et al. The Impact of Macro-and Micronutrients on Predicting Outcomes of Critically III Patients Requiring Continuous Renal Replacement Therapy. PLoS ONE. 2016; 11(6): e0156634.

(9) Cano NJM, Aparicio M, Brunori G, Carrero JJ, Cianciaruso B, Fiaccadori $E$, et al. ESPEN Guidelines on Parenteral Nutrition: adult renal failure. Clin Nutr. 2009; 28(4): 401-14.

(10) McClave SA, Taylor BE, Martindale RG, Warren MM, Johnson $D R$, Braunschweig $C$, et al. Guidelines for the Provision and Assessment of Nutrition Support Therapy in the Adult Critically III Patient: Society of Critical Care Medicine (SCCM) and American Society for Parenteral and Enteral Nutrition (A.S.P.E.N.). JPEN J Parenter Enteral Nutr. 2016; 40(2): 159-211. 